\author{
ANNALES \\ POLONICI MATHEMATICI \\ XXXII (1976)
}

\title{
On quasi-starlike functions
}

\author{
by R. W. Barnard (Lubbock, Texas)
}

\begin{abstract}
Let $S^{*}$ be the usual class of normalized starlike functions $F(z)$ on the unit disk $U=\{z:|z|<1\}$. If $g(z)$ is regular in $U$ and satisfies the condition $M F[g(z)]$ $=F(z), z \in U$, for some $F \in S^{*}$ and some positive number $M>1$, then $g$ is said to be in $G^{M}$. In Ann. Polon. Math. 20 (1968), p. 280-282 and ibidem 26 (1972), p. 175-197, I. Dziubiński defined the class $G^{M}$ and called $g$ in $G^{M}$ a quasi-starlike function. He raised the question of inclusion relations between $S^{*}$ and $G^{M}$ and asked if every bounded starlike function is quasi-starlike. We answer the question in the negative by exhibiting a bounded starlike function that is not quasi-starlike. We also show that if $F$ is either a strongly starlike function of order $1 / 2$ as defined by Brannan and Kirwan in J. London Math. Soc. (2) 1 (1969), p. 431-443, or if $F$ is a circularly symmetric function, then $g$ defined by $M F[g(z)]=F(z)$ is starlike. We also show that the $1 / 2$ is best possible in the sense that for every $\varepsilon, 0<\varepsilon<1 / 2$, there exists a strongly starlike function $f$ of order $\varepsilon+1 / 2$ such that the $g$ defined by $M f[g(z)]=f(z)$ is not starlike.
\end{abstract}

1. Introduction. Let $S$ denote the class of regular univalent functions $f(z)=z+a_{2} z^{2}+\ldots$ in the unit disk $U$. Let $S^{*}$ denote the subclass of $S$ of functions $f$ such that $f(U)$ is starlike with respect to the origin. We use starlike to mean starlike with respect to the origin. In [3] and [4] I. Dziubiński introduced the class of functions $\tilde{\mathcal{S}}_{M}^{*}$ that he called quasi-starlike. He defined for $M>1$,

$$
\tilde{S}_{M}^{*}=\left\{g: M f[g(z)]=f(z), f \in S^{*}, z \in U\right\},
$$

where $g$ is said to be generated by $f$. Then $M g(z)=z+\ldots$ is a normalized quasi-starlike function and is in S. In [4] Dziubiński posed the problem as to whether every starlike function bounded in $U$ is a normalized quasistarlike function. He also discussed the difficulty in obtaining conditions for a quasi-starlike function to be starlike. He stated that the difficulty arises because a quasi-starlike function can be easily constructed from any given starlike function.

In this note we give an example of a bounded starlike function that is not a normalized quasi-starlike function and give some sufficient conditions for a normalized quasi-starlike function to be starlike.

2. An example. Let $F$ be the bounded starlike function such that $F(U)$ is a disk minus two radial slits. The disk is centered at the origin. 
and of radius $M$, while the slits are non-vertical, non-horizontal and symmetric about the real axis. We will show that $F$ can not be a normalized quasi-starlike function. Assume to the contrary. Then there exists an $f$ in $\mathcal{S}^{*}$ and an $M>1$ such that $F(z)=M f^{-1}[f(z) / M]$. For any set $X$ let $f(X)=\{f(x): x \in X\}$. We first show that $f(U)$ must be a slit domain. Let $g(z)=F(z) / M$ with $F$ as defined above. Since $f \epsilon S^{*}, f[g(U)]=f(U) / M$ is a starlike domain and $f[g(U)]=f(U)-f\left(l_{1}\right) \cup f\left(l_{2}\right)$, where $M l_{1}$ and $M M_{2}$ are the symmetric, radial, linear slits in $F(U)$. Since $f[g(U)]$ is starlike, $f\left(l_{1}\right)$ and $f\left(l_{2}\right)$ must be radial slits. It now follows easily from the equation

$$
f(U)-f\left(l_{1}\right) \cup f\left(l_{2}\right)=\frac{f(U) !}{M}
$$

that $f(U)$ is the plane minus two radial slits.

From this geometric description, $f$ must assume the following form:

$$
f(z)=\frac{z}{\left(1-\sigma_{1} z\right)^{a}\left(1-\sigma_{2} z\right)^{2-a}}
$$

for some $a, 0<a<2$, and $\left|\sigma_{k}\right|=1, k=1,2$. Dziubinski showed in [4], Theorem 3, that the only time a function of the form (2) generates a quasistarlike function that is starlike is when $a=1$ and $\sigma_{k}=\exp i(-1)^{k-1} \theta$, $k=1,2$, for any $\theta \in(0, \pi)$. This would imply the two radial slits in $f(U)$ are opposing slits (i.e., their arguments differ by $\pi$ ). But this would force the slits in $F(U)$ to be opposing slits also. This contradicts the definition of $F$. Therefore $F$ is a bounded starlike function that is not a normalized quasi-starlike function.

3. Conditions for starlikeness. To establish these conditions we need the definitions of two subclasses of S. Jenkins stated in [6] that a domain $D$ is circularly symmetric with respect to the positive reals if every circle centred at the origin intersects $D$ in at most one arc $\gamma$ such that $\gamma$ is symmetric with respect to the positive reals. We say a function $f$ is in $\boldsymbol{F}$ if $f$ is in $S$ and $f(U)$ is circularly symmetric with respect to the positive reals. We will suppress the term "with respect to the positive reals". Also, in [1], Brannan and Kirwan defined the class of strongly starlike functions $\mathcal{S}^{*}(a)$, where, for given $a, 0 \leqslant a \leqslant 1, f \in \mathcal{S}^{*}(a)$, if and only if

$$
\left|\arg \frac{z f^{\prime}(z)}{f(z)}\right| \leqslant \frac{a \pi}{2}, \quad z \in U .
$$

The main theorem is as follows:

THEOREM. Let $F$ be in $\tilde{S}_{M}^{*}$ with $F$ defined by

$$
M f[F(z)]=f(z), \quad z \in U
$$


for $f \in \mathbb{S}^{*}$ and $M>1$. If either

(a) $f \in Y$, or

(b) $f \in S^{*}(a), 0 \leqslant a \leqslant 1 / 2$,

then $M F$ is in $S^{*}$. The $1 / 2$ in (b) is sharp.

Remark. The sharpness result of (b) is in the sense that for every $\varepsilon>0$ there exists a function $f_{0}$ in $\mathcal{S}^{*}(\varepsilon+1 / 2)$ that generates a function in $S_{M}^{*}$ that is not starlike for some $M=M(\varepsilon)$.

Proof. Take the logarithmic derivative of (4) with respect to $z$ and then multiply by $z$ to obtain:

$$
\frac{\left\{\frac{d}{d F} f[F(z)]\right\} z \frac{d}{d z} F(z)}{f[F(z)]}=\frac{z \frac{d}{d z} f(z)}{f(z)} .
$$

Let $w=F(z)$, where $|w|<1$ and let $f^{\prime}(w)=\frac{d f(w)}{d w}$. Then using (4) we have

$$
\frac{z F^{\prime}(z)}{F^{\prime}(z)}=\frac{f(w)}{w 0 f^{\prime}(w)} \frac{z f^{\prime}(z)}{f(z)} .
$$

Since $M F$ is starlike if and only if

$$
\left|\arg \frac{z F^{\prime}(z)}{F(z)}\right|<\frac{\pi}{2}, \quad z \in U
$$

we need only show that conditions (a) and (b) separately imply that

$$
\left|\arg \frac{z f^{\prime}(z)}{f(z)} \frac{f(w)}{w f^{\prime}(w)}\right|<\frac{\pi}{2}, \quad z \in U .
$$

To prove part (a) of the theorem, consider an $f$ in $\boldsymbol{Y}$. Let $P(\zeta)$ $=\zeta f^{\prime}(\zeta) / f(\zeta)$ for any $\zeta \epsilon U$. It follows from a result of Jenkins in [6] that if $f \in Y$, then either $f$ is the identity function or

$$
\begin{aligned}
& \operatorname{Im}\{z\} \operatorname{Im}\{f(z)\} \geqslant 0, \\
& \operatorname{Im}\{z\} \operatorname{Im}\{P(z)\} \geqslant 0,
\end{aligned}
$$

The case when $f$ is the identity follows immediately, so assume $f$ is not the identity. Consider the two cases, $\operatorname{Im}\{z\} \geqslant 0$ and $\operatorname{Im}\{z\} \leqslant 0$. When $\operatorname{Im}\{z\} \geqslant 0$, since $F(z)=w$ is defined by (4), we have that $\operatorname{Im}\{w\}$ $\geqslant 0$. Hence, since $f \in Y$, property (7) assures that $\operatorname{Im}\{P(z)\} \geqslant 0$ and $\operatorname{Im}\{P(w)\} \geqslant 0$. Thus, since $\operatorname{Re}\{P(z)\}$ and $\operatorname{Re}\{P(w)\}$ are positive from the starlikeness of $f$, we have $0 \leqslant \arg P(z)<\pi / 2$ and $0 \leqslant \arg P(w)<\pi / 2$ for $\operatorname{Inn}\{z\} \geqslant 0$. Hence $|\arg [P(z) \mid P(w)]|=|\arg P(z)-\arg P(w)|=|| \arg P(z) \mid-$ $-|\arg P(w)| \mid \leqslant \max [\arg P(w), \arg P(z)]<\pi / 2$. A corresponding argu- 
ment will show that if $\operatorname{Im}\{z\} \leqslant 0$, then $|\arg [P(z) \mid P(w)]|<\pi / 2$. Therefore (6) follows. (3),

For part (b) of the theorem, let $f \in S^{*}(a)$ for $0 \leqslant \alpha \leqslant 1 / 2$. Then using

$$
\left|\arg \frac{z f^{\prime}(z)}{f(z)} \cdot \frac{f(w)}{w f^{\prime}(w)}\right| \leqslant\left|\arg \frac{z f^{\prime}(z)}{f(z)}\right|+\left|\arg \frac{w f^{\prime}(w)}{f(w)}\right| \leqslant \frac{\pi}{4}+\frac{\pi}{4}=\frac{\pi}{2}
$$

Thus (6) follows.

To verify the sharpness result, we show that for every $\varepsilon>0$ there exists a function in $\mathcal{S}^{*}(\varepsilon+1 / 2)$ that generates a function in $\tilde{S}_{M}^{*}$ that is not starlike for some $M>1$. Let $D(a)$ denote the pie shaped convex domain bounded by the left half of the unit circle $A \widehat{C}$ and two line segments $\overline{A B}$ and $\overline{B C}$ having angles of inclination with the positive real axis $\pi \pm(1-a) \pi / 2(0<a<1)$, respectively. Let $g$ be the corresponding mapping function such that $g(U)=D(a)$ with $g(0)=0$ and $g^{\prime}(0)>0$. It is clear that $g$ extends to a continuous function on the closure of $U$ that is differentiable on $U$ except at the preimages of the three corners of $D(\alpha)$. We denote the extended function as $g$ also. Note the function $g(z)=a_{1} z+\ldots$, where $a_{1}$ is positive, is such that $\frac{1}{a_{1}} g$ is in $S^{*}(\alpha)$. Given an $\varepsilon>0$, let $a_{s}=1 / 2+\varepsilon / 2$. Ohoose an $M>1$ such that $M g\left(w_{0}\right)=g\left(z_{0}\right)$ defines a $w_{0}$ with $\arg \left[w_{0} g^{\prime}\left(w_{0}\right) / g\left(w_{0}\right)\right]=(1 / 2+\varepsilon / 8) \pi / 2 . M$ can be chosen in this manner since $w_{0} \rightarrow z_{0}$ as $M \rightarrow 1$ and $\arg \left[w_{0} g^{\prime}\left(w_{0}\right) / g\left(w_{0}\right)\right]$ increases to $\arg \left[z_{0} g^{\prime}\left(z_{0}\right) / g\left(z_{0}\right)\right]=(1 / 2+\varepsilon / 4) \pi / 2$. Now we shall construct a sequence of domains which converge to $D\left(a_{s}\right)$ and such that their corresponding mapping functions will converge uniformly on compact subsets of $U$ to $g$. Let $n$ be a large positive integer. Consider the domain bounded by an arc ${\widetilde{A_{n}}}_{n}$ that is the left half of a circle centered at the origin with $\operatorname{Im}\left\{A_{n}\right\}$ $>0$, the line segment $\overline{C_{n} B_{n}}$ parallel to $\overline{C B}$, a line segment $\overline{E_{n} D_{n}}$ of length $1 / n$, parallel to $\overline{C B}$ and having $g\left(z_{0}\right)$ as its midpoint, and the line segments $\overline{A_{n} D_{n}}$ and $\overline{E_{n} B_{n}}$ that are parallel to $\overline{A B}$ and that complete the boundary of this simply connected domain. Denote this domain as $G_{n}$ with corresponding mapping function $g_{n}$ such that $g_{n}(U)=G_{n}$. It is clear that as $n \rightarrow \infty, G_{n}$ converges to $D\left(a_{\varepsilon}\right)$ in the sense of Carathédory. From the Carathédory convergence theorem [5] $g_{n}$ converges to $g$ uniformly on compact subsets of $U$. For each $n$, let $z_{n}$ be the point on the unit circle such that $g_{n}\left(z_{n}\right)=g\left(z_{0}\right)$. From the construction of $g_{n}$ we have $\arg \left[z_{n} g_{n}^{\prime}\left(z_{n}\right) / g_{n}\left(z_{n}\right)\right]=-(1 / 2+3 \varepsilon / 4) \pi / 2$ for each $n$. Let $w_{n}$ be the point in $U$ such that $M g_{n}\left(w_{n}\right)=g_{n}\left(z_{n}\right)=g\left(z_{0}\right)$. From the uniform convergence of $g_{n}$ to $g$ on compact subsets of $U$ we have that $w_{n} \rightarrow w_{0}$ as $n \rightarrow \infty$, while Weierstrass' Theorem assures that $\arg \left[w_{n} g_{n}^{\prime}\left(w_{n}\right) / g_{n}\left(w_{n}\right)\right]$ approaches $\arg \left[w_{0} g^{\prime}\left(w_{0}\right) / g\left(w_{0}\right)\right]$. Thus there exists an integer $N$ such that $g_{N}$ is in 
$S^{*}(\varepsilon+1 / 2)$ while

$$
\begin{aligned}
\left|\arg \frac{z_{N} g_{N}^{\prime}\left(z_{N}\right)}{g\left(z_{N}\right)}-\arg \frac{w_{N} g_{N}^{\prime}\left(w_{N}\right)}{g_{N}\left(w_{N}\right)}\right| & \geqslant\left|-\left(\frac{1}{2}+\frac{3 \varepsilon}{4}\right) \frac{\pi}{2}-\left(\frac{1}{2}+\frac{\varepsilon}{8}\right) \frac{\pi}{2}\right| \\
& =\left(1+\frac{7 \varepsilon}{8}\right) \frac{\pi}{2}>\frac{\pi}{2} .
\end{aligned}
$$

Therefore it follows from (6) that $g_{N}$ generates a quasi-starlike function that is not starlike. This completes the proof of the theorem.

Let $C(B)$ denote the subclass of $S$ of function $f$ such that $f(U)$ is convex and $|f(z)| \leqslant B, z \in U$. The author can show by long, but straightforward, arguments that there exist finite $B$ 's for which there are functions in $C(B)$ that generate quasi-starlike functions that are not starlike. Thus there exists a finite $B_{0}$ that is the supremum of all $B$ 's such that if $f \in C(B)$, then $f$ generates a quasi-starlike function that is starlike for all $M>1$. The following corollary gives a lower bound for $B_{0}$.

CoRoLla RY. If $f \in C(B)$ with $B \leqslant \sqrt{32 / 27}$, then $f$ generates a quasistarlike function that is starlike for all $M>1$.

Proof. In [2] Brannan and Kirwan proved that if $f \in C(B)$, then $f \in S^{*}(\alpha)$ with

$$
a=1-\frac{2}{\pi} \arcsin [\delta(B) / B]
$$

where $\delta(B)$ denotes the Koebe constant for $C(B)$ (i.e., the radius of the largest open disk centered at the origin and contained in the image of $U$ under every function in $C(B)$ for a fixed $B$ ). The value of $\delta(B)$ has been determined by Krzyż in [7] to satisfy

$$
\delta(B)=B \sin \theta,
$$

where $\theta$ is the unique solution of the equation,

$$
(\pi+2 \theta) \sin \frac{4 \pi \theta}{\pi+2 \theta}=2 \pi B^{-1} \cos \theta .
$$

The result follows by letting $\alpha=1 / 2$ in (8) and then solving for $B$ in (9) and (10).

\section{References}

[1] D. Brannan and W. Kirwan, On some classes of bounded univalent functions, J. London Math. Soc. (2) 1 (1969), p. 431-443.

[2] - - The Maclaurin coefficients of bounded convex functions, Bull. London Soc. (2) (1970), p. 159-164. 
[3] I. Dziubiński, Quasi-starlike functions, Bull. Acad. Polon. Sci. 16 (6) (1968), p. $477-479$.

[4] - Quasi-starlike functions, Ann. Polon. Math. 26 (1972), p. 175-197.

[5] G. M. Goluzin, Geometric theory of functions of a complex variable, Trans. Math. Mono. Amer. Math. Soc. 26 (1969).

[6] J. A. Jenkins, On circularly symmetric funotions, Proc. Amer. Math. Soc. $\theta$ (1958), p. 82-87.

[7] J. Krzyi, Distortion theorems for bounded convex funotions, Bull. Acad. Polon. Sci. 8 (9) (1960), p. 625-627.

Reçu par la Rédaction le 25.7.1974

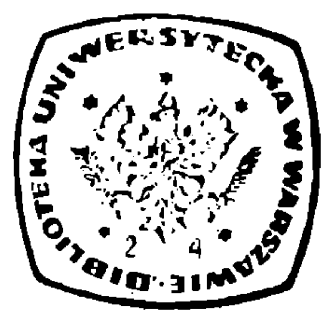

